\title{
Recenser et évaluer les pratiques agricoles qui stockent le carbone des sols, premier pas vers une agriculture à faible impact en Guyane
}

\author{
Amandine Courte ${ }^{1}$, Nathalie Cialdella ${ }^{2,4}$, Alexandre Muller ${ }^{3,4}$, Vincent Blanfort ${ }^{3,4}$, Jean-Luc Bochu ${ }^{5}$ \\ et Michel Brossard ${ }^{1, *}$ \\ ${ }^{1}$ IRD, UMR 210 Eco\&Sols, BP 90165, 97323 Cayenne Cedex, France \\ 2 CIRAD, UMR INNOVATION, Campus agronomique, BP 701, 97387 Kourou Cedex, Guyane française \\ 3 CIRAD, UMR SELMET, Campus international de Baillarguet, 34398 Montpellier Cedex 5, France \\ ${ }^{4}$ Univ. Montpellier, Montpellier, France \\ 5 SOLAGRO, CS 27608, 31076 Toulouse Cedex 3, France
}

\begin{abstract}
Résumé - En Guyane, les questions environnementales et le besoin croissant en énergie et produits alimentaires liés à la croissance démographique exercent une pression accrue sur les ressources naturelles par le changement d'affectation des sols qu'ils engendrent. Des agriculteurs testent cependant des pratiques à faible impact conciliant objectifs de production et respect de l'environnement. Une prospection dans les systèmes de production agricoles guyanais, basée sur des enquêtes de terrain, a permis la description de ces pratiques; parmi celles-ci, les pratiques à faible impact sur la dynamique du carbone ont été sélectionnées dans des systèmes maraîchers et arboricoles, pour des mesures spécifiques des stocks de carbone des sols. En Guyane, la pratique de la défriche est toujours active afin d'étendre les surfaces agricoles utiles. Cela induit des pertes de carbone importantes. Nous cherchons donc à identifier des pratiques qui permettent de compenser ces pertes. La place du sol dans les prises de décision des agriculteurs a été appréhendée et des mesures spécifiques des stocks de carbone des sols ont complété l'enquête, pour mieux comprendre les logiques des pratiques mises en œuvre et en cerner les effets. Nos résultats montrent que les agriculteurs maitrisent «l'objet sol» dans ses composantes physiques; la matière organique est un indicateur reconnu qui conditionne l'organisation des cultures sur l'exploitation. En Guyane, les agriculteurs mobilisant des pratiques à faible impact ont des systèmes de production efficients au niveau économique et environnemental (carbone et gaz à effet de serre).
\end{abstract}

Mots clés : système de culture / Amazonie / petite agriculture tropicale

\begin{abstract}
Identification and evaluation of agricultural practices that store soil carbon, a first step towards low impact agriculture. In French Guiana, the general environmental context and the growing need for energy and food products, linked to population growth, are responsible for increasing pressure on natural resources through land use changes. Low impact practices are emerging in the aim of reconciling production and environmental objectives. In French Guiana slash and burn practices are still used to increase agricultural area, and lead to a large loss of carbon. A field survey was carried out in order to understand the place of soil in farmers' choices, and to obtain a description of production systems and practices, focusing on identifying those with low impact on carbon dynamics. This step was realized through specific soil carbon stock measurements. Our results show that the "soil object" is mastered by farmers in terms of physical properties, and organic matter is a recognized indicator which is used to inform spatial management of farming systems. Farmers mobilizing low-impact practices have efficient production systems for their economic and environmental benefits.
\end{abstract}

Keywords: agricultural system / Amazonia / small tropical agriculture

\footnotetext{
*Auteur de correspondance : michel.brossard@ird.fr
} 


\section{Introduction}

Le développement de l'agriculture en zone intertropicale concerne $130000 \mathrm{~km}^{2}$.an ${ }^{-1}$ et engendre des émissions de $\mathrm{CO}_{2}$ suite aux pratiques de la défriche forestière (GIEC, 2019), tout en étant atteint par les effets du changement climatique (CC). Le sol est un système qui tient une place centrale dans la régulation des écosystèmes agricoles et forestiers (Baveye et al., 2016; FAO, 2018), du changement climatique (Lal et al., 2007) et de la sécurité alimentaire (McBratney et al., 2014). En étant à la fois un lieu de stockage du carbone (C) et une source d'émission de gaz à effet de serre (GES), les sols sont l'interface de phénomènes accentués lors des changements d'usage, défriches et mises en culture. Ces modifications accélèrent l'oxydation de la matière organique, et donc altèrent le potentiel de fertilité senso latu des sols (FAO, 2015).

En Guyane, les changements d'usage des terres représentent $43 \%$ des émissions de GES du territoire (GEC, 2016). Les surfaces $(<10$ ha par producteur) ne suffisent pas à alimenter la population; une grande partie des produits alimentaires sont importés; de plus, l'agriculture est peu rémunératrice (IEDOM, 2019). L'agriculture guyanaise doit se développer afin d'assurer l'alimentation d'une population croissante, qui a doublé en 20 ans (INSEE, 2018). Elle doit garantir un meilleur niveau de vie pour les familles, faire face à des contraintes agronomiques et environnementales fortes (Renoux et al., 2003), tout en affichant un impact carbone le plus réduit possible. Une agriculture moins traditionnelle s'est développée dans les années 1960 avec le Plan vert (Piantoni, 2009), mais reste encore méconnue. Le développement de l'agriculture entraîne sur ce territoire des changements d'usage des terres qui induisent des pertes de $\mathrm{C}$ dans la biomasse, mais aussi dans le sol. Dans ce cadre, les agriculteurs doivent mobiliser des pratiques qui puissent maintenir, voire reconstituer, les niveaux du stock organique de carbone (SOC). Ce sont ces pratiques que nous proposons de désigner comme à faible impact sur le carbone et qui nous intéressent dans cette étude.

À l'instar de réflexions similaires sous d'autres latitudes (Razafimbelo et al., 2018), l'objectif est de s'intéresser aux pratiques à faible impact sur le carbone, dans une réflexion systémique qui prenne en compte la connaissance des systèmes de production. Aussi, nous avons caractérisé les systèmes de production(SP) de la petite agriculture, les pratiques usitées par les agriculteurs et la place du sol dans les prises de décision des agriculteurs et dans la dynamique de gestion des exploitations. Cela permet de savoir si les pratiques à faible impact existent, d'en faire un premier inventaire et de proposer un référentiel. Nous proposons ici une identification et une évaluation de pratiques agricoles vertueuses en termes d'impact sur le climat par le biais d'une vision systémique. À partir de cette analyse des critères agraires, nous avons sélectionné des sites représentatifs pour évaluer les stocks organiques de carbone dans cette petite agriculture. Des mesures de stock ont donc été réalisées de manière synchrone sur ces sites forestiers qui constituent des $\mathrm{T}_{0}$ (situation à l'état initial) et sur des parcelles agricoles; ainsi, nous discutons ces pratiques et l'état des stocks de C agricole pour déterminer le devenir du carbone après mise en culture.

\section{Démarche méthodologique}

\subsection{Les enquêtes}

Les enquêtes ont été faites auprès de 69 agriculteurs (soit $1,2 \%$ des exploitants de Guyane) choisis aléatoirement le long du littoral (Fig. 1) et ont permis de décrire les systèmes de production et les pratiques. Les exploitations se situent dans des zones désignées par les services de l'Etat comme «agricoles prioritaires » ou « occupées ». Les premières (prioritaires) sont vouées à être défrichées à l'horizon 2030 pour y développer l'agriculture tandis que les secondes (occupées) sont des zones où se concentre déjà l'activité agricole. Ces exploitations se localisent au Nord de la Guyane, où se concentrent les activités humaines sur 16 sous-types de sols répertoriés dans la carte pédologique. Nous nous focalisons sur l'étude des systèmes maraîchers et arboricoles (séparés ou mélangés au sein d'une exploitation), qui représentent $70 \%$ des systèmes de production guyanais ; les systèmes pâturés sont quant à eux mieux connus et traités dans d'autres programmes.

Nous nous sommes placés dans une dynamique de connaissance de la diversité des systèmes de production pour identifier les modes d'actions efficaces (Cochet et Devienne, 2006) conciliant préservation environnementale et fertilité des sols. En effet, l'objectif est de mettre en avant des pratiques locales et d'en illustrer les impacts sur le stockage du carbone. Les entretiens semi-directifs (Imbert, 2010; Olivier de Sardan, 1995a) ont été conduits à partir d'une grille d'entretien construite sur la base de connaissances expertes, afin d'obtenir des données sur : i) l'organisation de la structure agricole, ii) le parcours de vie de l'exploitant, iii) les aspects économiques, iv) les pratiques agricoles. L'arrêt de l'échantillonnage s'est fait une fois arrivé à saturation des données (Olivier de Sardan, 1995b). La typologie a été construite à dire d'experts (Landais, 1998).

\subsection{La place du sol dans la prise de décision des exploitants}

Nous sommes retournés chez 45 des 69 agriculteurs interrogés pour obtenir des compléments de données sur la place que prend le sol dans leurs prises de décision et sur leur niveau de connaissance de l'objet sol.

La perception du sol a été traitée sur la base des questions suivantes : Quels sont les principaux freins à la gestion de votre exploitation? Qu'est-ce qu'un bon sol selon vous? Quels indicateurs vous permettent de mesurer ou d'évaluer la qualité de votre sol? Quelles pratiques vous semblent les plus efficaces pour améliorer ou conserver la fertilité du sol? Quelles sont les contraintes à leur mise en place?

Nous avons cherché à déterminer la place que joue le sol dans la logique d'action de l'agriculteur. L'occurrence des réponses a été traitée et a permis de catégoriser les freins au développement agricole, caractériser leur nature et les classer selon trois niveaux d'importance (agriculteurs faiblement impactés, impactés et fortement impactés). L'absence de réponse à une ou plusieurs questions a aussi été prise en compte lorsqu'elle n'exprimait pas une volonté de ne pas participer à l'étude, mais prenait les formes suivantes : «cela ne m'intéresse pas», «je ne me sens pas concerné» ou «je ne sais pas ». 


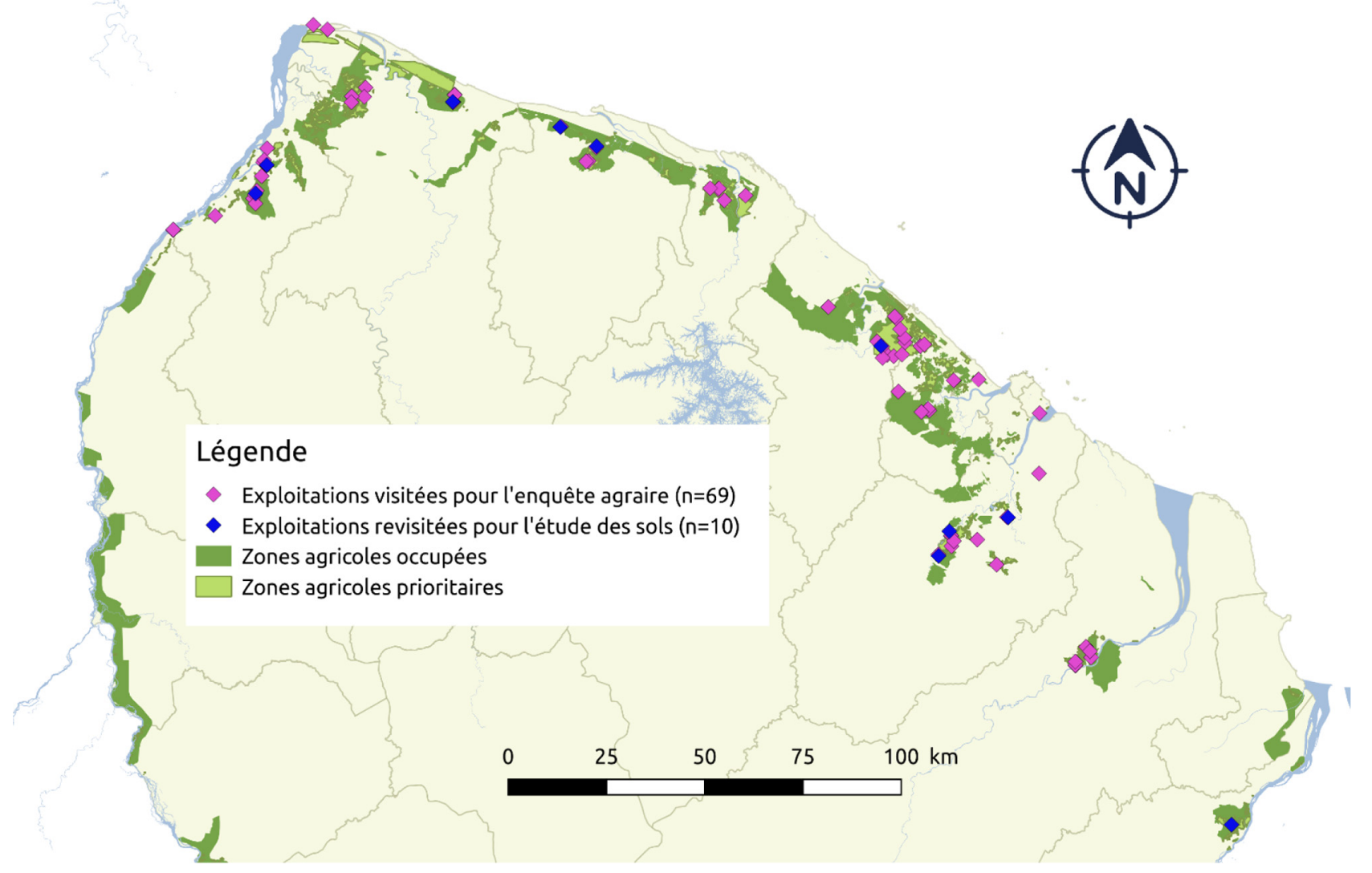

Fig. 1. Situation géographique des exploitations étudiées et des sites où les stocks de carbone des sols ont été prélevés en milieu agricole. Fig. 1. Map of farms surveyed and agricultural places where soil carbon stocks have been measured.

\section{3 Évaluer le carbone et l'effet des pratiques}

$\mathrm{Au}$ total, 31 parcelles agricoles (Fig. 1), situées chez 10 agriculteurs représentatifs de la typologie (en termes de pratique et de gestion) et dans une station expérimentale, ont été sélectionnées pour réaliser des prélèvements de sol ( 5 parcelles avec usage de pratiques à fort intrants, 20 avec usage de pratiques à faible impact et 6 avec usage de pratiques traditionnelles). Plusieurs parcelles ont été échantillonnées par agriculteur lorsque les sols ou les pratiques variaient au sein d'une même exploitation. De nombreuses parcelles ont été étudiées dans des systèmes à faible impact, puisque ces pratiques nous intéressent particulièrement dans cette étude et que ce sont les pratiques les plus diversifiées. Les mesures du stock organique de carbone agricole et forestier sont classiques (FAO, 2019; RMQS : www.gissol.fr) et s'appuient sur des mesures de densité apparente. Ici, les mesures sont réalisées sur des échantillons composites pour les couches $0-0,3$ et $0,3-$ $0,5 \mathrm{~m}$ de sol, en prenant en compte, le cas échéant, l'organisation spatiale des parcelles (rang et inter-rang). Les comparaisons entre les SOC à $\mathrm{T}_{0}$ avant défriche et les SOC agricoles à $T_{+n}$ (entre 2 et 30 ans) ont été effectuées à masse de sol équivalente pour la couche considérée. Les échantillons $\mathrm{T}_{0}$ ont été choisis à dire d'experts en fonction des particularités pédologiques observées sur le terrain et prélevés au voisinage des sites. Nous étudions donc des pratiques mises en place à des pas de temps différents. Le bilan des émissions des gaz à effet de serre a été effectué chez 9 de ces 10 agriculteurs en s'appuyant sur l'outil ACT-DOM. L'outil est en cours d'adaptation sur le territoire guyanais et permet d'établir des diagnostics Energie et GES à l'échelle de la ferme (Dallaporta et al., 2016). Pour utiliser cet outil, nous avons collecté des données quantitatives (intrants, surfaces couvertes, densité du plastique des serres, quantités produites et exportées, etc.). Ainsi, nous obtenons pour chaque exploitation ses émissions en teq $\mathrm{CO}_{2} \cdot \mathrm{ha}^{-1} \cdot \mathrm{an}^{-1}$. Ce bilan n'a pas pu être réalisé chez des agriculteurs aux pratiques traditionnelles (abattis-brûlis itinérants), car les données quantitatives sont absentes. Cela constitue une limite pour l'obtention de bilans carbone fins.

\section{Résultats}

\subsection{Les systèmes de production observés}

Les systèmes de production observés se distinguent dans un premier temps par les pratiques (à faible impact, à forts intrants et traditionnelles) et dans un second par l'objectif de production (commercialisation, transformation, vivrier) (Fig. 2).

\subsubsection{Les systèmes à faible impact (30 exploitations)}

Dans ce groupe, nous retrouvons des exploitations récentes (maraîchage et arboriculture) en phase d'installation (24\%) et 

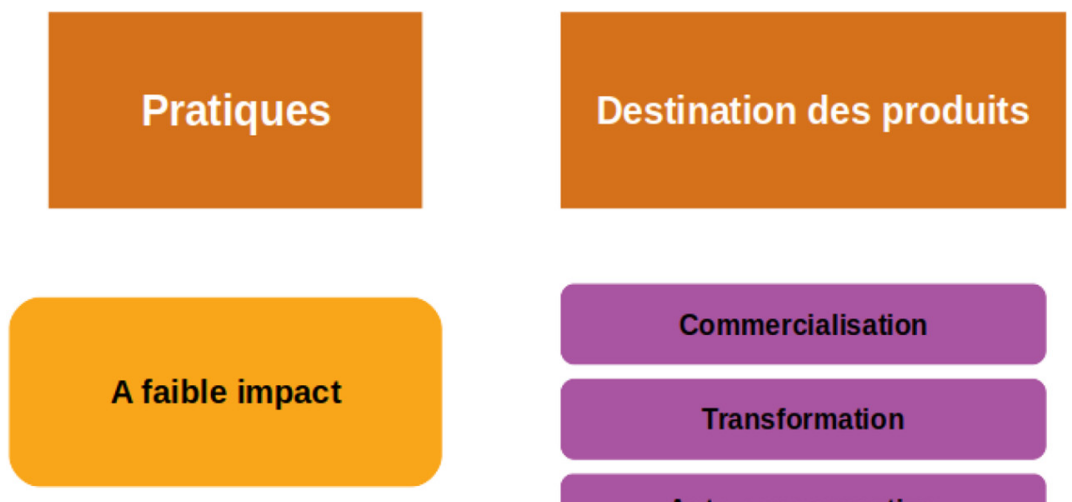

Auto-consommation

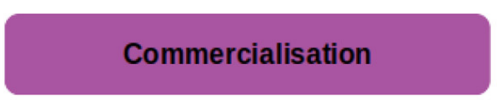

A forts intrants
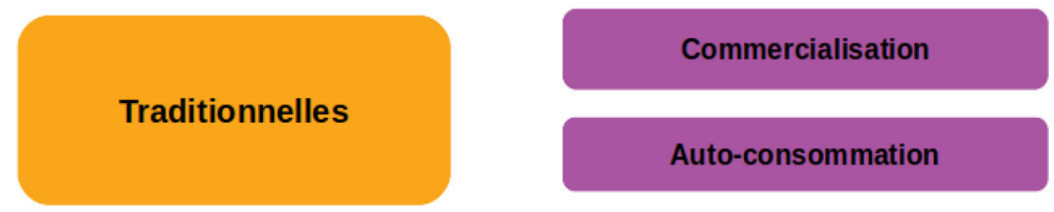

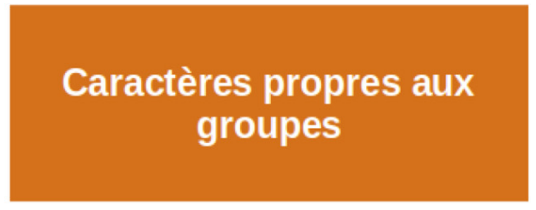

1.1.1 Nouvelles exploitations à aspiration biologique

1.1.2 Les exploitations stables certifiées AB. 1.1.3 Pluri-Atelier

\subsection{Agriculture AB avec vocation de transformation}

1.3 Agriculture vivrière conservatoire

Fig. 2. Typologie des exploitations.

Fig. 2. Farm typology.

des exploitations plus anciennes issues d'un héritage, où le système de production est bien en place. Le projet partagé de ces exploitants est d'optimiser leur production dans une démarche de respect de l'environnement; $30 \%$ d'entre eux sont d'anciens agriculteurs conventionnels (faisant usage de pratiques à forts intrants) qui ont réalisé une conversion vers l'agriculture biologique. La prise de décision est fonction de valeurs et n'est pas dictée directement par un objectif d'augmentation du chiffre d'affaire ou de la production. Les agriculteurs y développent de nouvelles pratiques avec une gestion plus organique et sans produits chimiques. Ils travaillent essentiellement manuellement et réutilisent les sous-produits de leur exploitation ; $10 \%$ produisent et utilisent du bois raméal fragmenté (BRF) issu de la défriche de leur sous-bois. Si les ressources financières pour engager un salarié le permettent, l'entretien des parcelles est alors partiellement délégué. Lors des périodes de récolte, la famille ou les amis viennent soutenir l'activité. Leur production de fruits ou légumes est vendue sur les marchés, directement depuis l'exploitation, à la coopérative locale ou dans de grandes et moyennes surfaces. Sont comprises dans ces systèmes les exploitations en polyculture-élevage et des transformateurs (commercialisation d'un unique produit transformé); l'activité d'élevage ou de transformation y représente une grande partie du revenu, du temps ou de l'investissement. En polycultureélevage, l'atelier animal soutient économiquement l'atelier végétal. Les parcelles sont organisées dans l'espace et les pratiques orientées vers la fertilité du sol : utilisation de fumier, couvert végétal, rotation, bois raméal fragmenté. Dans les systèmes de production dédiés à la transformation, la production des produits transformés représente l'intégralité du revenu. Lorsque la production ne suffit pas à alimenter l'atelier de transformation, l'approvisionnement est complémenté par des achats extérieurs. Dans les systèmes de production avec un objectif d'autoconsommation, les agriculteurs bénéficient souvent d'un revenu supplémentaire à la simple vente des surplus de leur production, leur permettant d'investir dans leur activité agricole. Les pratiques agricoles sont réfléchies, se veulent à faible impact et particulièrement diverses et les caractéristiques du sol sont maîtrisées. Ces agriculteurs n'ont pas de grandes surfaces (moyenne de 8,5 ha) et leurs parcelles se situent à proximité de l'habitation.

\subsubsection{Les systèmes à forts intrants (19 exploitations)}

Dans ces exploitations mécanisées, le recours aux intrants chimiques est systématique et s'accompagne rarement d'apport de matière organique fraîche. Le temps de travail est partagé entre maraîchage et arboriculture, les parcelles sont généralement monospécifiques (ananas, concombre, banane, agrumes, etc.). Le revenu est exclusivement issu de l'activité de l'exploitation et est suffisant pour lui permettre de se développer. 
Tableau 1. Hiérarchisation des freins au développement agricole.

Table 1. Impediments to farming development.

\begin{tabular}{llllll}
\hline Système de production & Pédoclimat & Difficulté administrative & facilité à disposer des aides & Enclavement & Manque de filière \\
\hline À forts intrants & + & + & +++ & + & ++ \\
À faible impact & ++ & + & ++ & ++ & ++ \\
Traditionnel, vivrier & +++ & + & - & + & - \\
\hline
\end{tabular}

- Pas impacté.

+ faiblement impacté.

++ Impacté.

+++ Fortement impacté.

\subsubsection{Les systèmes traditionnels ( 20 exploitations)}

La particularité de ces systèmes de production réside dans la difficulté d'accès au foncier. Le premier groupe d'exploitations $(40 \%)$ correspond à des zones d'habitation traditionnelle très ancienne, mais sans titre de propriété ni statut agricole. L'agriculture est plutôt vivrière et ne représente pas l'activité principale. Ces exploitants n'ont pas les moyens d'investir dans l'agriculture. Le second groupe (60\%) qui se distingue dans ces systèmes de production est formé par des agriculteurs nouvellement installés qui perçoivent les aides et subventions sectorielles et qui cherchent à diversifier leurs productions, dans des circuits courts ou sur les marchés locaux et grâce à un statut professionnel agricole reconnu. Les exploitations se situent sur des terrains contraignants, aux sols sableux ou aux pentes fortes. Les surfaces cultivées sont petites $(<2 \mathrm{ha})$ et la production manuelle plutôt diversifiée: tubercules et fruits. Dans la moitié des cas, il s'agit d'abattis-brûlis avec une tendance à la diminution des surfaces cultivées. L'utilisation d'intrants est occasionnelle en fonction de la trésorerie disponible. Des planches de maraîchage, constituées d'un mélange de terre et de compost, leur permettent de pallier les contraintes des sols peu fertiles. Les techniques utilisées proviennent des acquis familiaux.

\subsubsection{Les freins rencontrés par les systèmes de production}

Nos résultats montrent que les agriculteurs les plus efficients sur le plan économique, aussi bien dans les "systèmes de production à forts intrants» que dans les «systèmes de production à faible impact», arrivent à organiser, et parfois à gérer eux-mêmes, la commercialisation de leur production grâce à une main-d'œuvre familiale importante dans le cas des « SP à forts intrants », ou grâce au recours à des marchés de niche pour les «SP à faible impact». Plusieurs freins à l'agriculture et au développement de pratiques à faible impact sont mis en évidence (Tab. 1). La lourdeur des démarches administratives, le manque d'instituts techniques et de filières structurées est manifeste. L'enclavement géographique, les terrains isolés difficiles d'accès compliquent la commercialisation; or la vente sur les marchés urbains constitue une des seules options rentables. Les conditions pédoclimatiques contraignantes sont systématiquement citées parmi les difficultés, même si elles sont moins importantes dans les systèmes de production à forts intrants. La saison des pluies, avec des précipitations de 2000 à $4000 \mathrm{~mm}$. $\mathrm{an}^{-1}$, est responsable d'excès d'eau et de maladies, et l'intensité des pluies favorise le ruissellement, puis l'érosion des sols. La couche supérieure du sol où est localisée la matière organique est en général peu épaisse, les sols sont naturellement acides, souvent carencés en calcium, ce qui nécessite des amendements calciques réguliers et coûteux. La variabilité des sols sur une même parcelle rend également difficile la gestion des cultures.

\subsection{Description de la diversité de pratiques}

On distingue un large panel de pratiques (Tab. 2). Les plus répandues, présentes chez $42 \%$ des agriculteurs interrogés, que nous nommerons «à forts intrants» par commodité, consistent en l'usage d'intrants chimiques pour la gestion des ravageurs et des maladies, des adventices et pour la fertilisation. Ces pratiques sont mobilisées dans les systèmes de production à forts intrants et ne sont pas détaillées ici, celles qui nous intéressent le plus étant celles à faible impact.

Sur la population étudiée, $51 \%$ des agriculteurs mettent en place au moins une pratique de conservation ayant pour but d'augmenter la fertilité du sol et limiter les émissions de GES. Ces pratiques, décrites ci-après, peuvent-être associées pour un effet combiné :

- utilisation des débris de fauche et de paillis $(20 \%$ des agriculteurs) apportés aux pieds des plants, parfois constitués par le broyage des recrus forestiers ou d'espèces volontairement plantées (légumineuses arbustives locales) et présentes en bordure de parcelle. Cependant, les débris de fauche sont souvent laissés sur place et non rassemblés au pied des plants, cette étape demandant un effort de travail trop conséquent;

- implantation de plantes de couverture vivantes $(7 \%$ des agriculteurs). Dans la moitié des cas, l'espèce implantée n'est pas connue. Elle est alors choisie pour sa faible hauteur et son pouvoir couvrant qui permettent de limiter l'entretien. L'autre moitié des agriculteurs choisit l'espèce à implanter - légumineuses ou herbes plus touffues (Cymbopogon sp, Bracharia sp) - pour l'apport d'azote ou pour l'apport de biomasse;

- association de cultures (22\% des agriculteurs) avec des mélanges d'espèces, comme ananas/banane, ou des systèmes agroforestiers plus complexes où arbres et cultures sont étroitement mélangés. Des rotations avec insertion de légumineuses existent également;

- constitution de buttes (9\% des agriculteurs) avec concentration de la matière organique. Ces buttes sont généralement décalées dans l'inter-rang tous les trois ans pour garder une parcelle relativement fertile; 
Tableau 2. Occurrence (\%) des réponses des exploitants pour les différents modes de gestion et pratiques agricoles.

Table 2. Occurrence (\%) of farmers' responses for different modes of management and farming practices.

\begin{tabular}{llr}
\hline Gestion des ravageurs/maladies & Aucune & 43 \\
& Organique & 34 \\
& Chimique & 23 \\
& Aucune & 7 \\
Gestion de la fertilité & Organique & 51 \\
& Chimique & 42 \\
& Aucune & 13 \\
Gestion des adventices & Organique & 57 \\
& Chimique & 30 \\
Jachère & Aucune & 73 \\
& 0-7 ans & 18 \\
Irrigation & + de 7 ans & 9 \\
& Oui & 57 \\
Paillis & Non & 43 \\
& Oui & 20 \\
Plantes de couverture & Non & 80 \\
& Oui & 7 \\
& Non & 93 \\
Mode de défriche $*$ & Brûlis + bulldozer & 18 \\
& Brûlis & 60 \\
Utilisation de «Terra preta » & Bulldozer & 12 \\
Association d'espèces & BRF & 10 \\
Buttes & Oui & 5 \\
& Non & 95 \\
& Oui & 22 \\
& Non & 78 \\
& Oui & \\
& Non &
\end{tabular}

*Brûlis + bulldozer : utilisation combinée des deux modes de défriche. Le feu permet un éclaircissement du sous-bois avant le passage du bulldozer pour coucher les bois restants et procéder au débardage.

Brûlis: Bois entièrement brulés sur la parcelle.

Bulldozer: Bois couchés et débardés de manière mécanique.

BRF : le sous-bois et les houppiers sont broyés et constituent un mulch avant le passage d'engins légers pour l'exploitation des troncs $>20 \mathrm{~cm}$ de diamètre.

- jachère de durée variable ( $27 \%$ des agriculteurs). La parcelle est mise en repos pendant 2 ou 3 ans, voire pendant plus de 7 ans, pour permettre la croissance du recru forestier, qui est ensuite brûlé avant de réinvestir la parcelle;

- confection d'un compost amélioré (5\% des agriculteurs). Un mélange de charbon, débris végétaux et fumier est constitué pour faire une «terre noire» inspirée des sols amérindiens précolombiens (terra preta). Certains peuvent aller jusqu'à la réalisation de charbon artisanal pour confectionner ce mélange, ce qui questionne le bilan GES d'une telle pratique.

\subsection{La perception des sols par les agriculteurs}

Les agriculteurs observent principalement leurs sols à travers la texture. Ils distinguent : «les sols rouges argileux », «les sols argileux mais un peu plus sableux», «les sols à dominante de sables» (ferralitisols argileux et argilo-sableux) et «les sols à sables blancs» (fluvisols, podzosols). Le drainage est noté dans la différenciation des sols, ce qui va déterminer l'emplacement des différentes cultures: «sols argileux drainants et trop secs en saison sèche», "sols hydromorphes » ou «gorgés d'eau en saison des pluies ». La présence d'éléments grossiers est souvent citée comme élément favorisant le drainage, même si aucune différence n'est évoquée quant à la nature et la taille de ces éléments (cailloux et gravillons ferrugineux, quartz...). Le travail du sol étant souvent concentré sur de petites surfaces peu mécanisées, graviers et cailloux sont souvent considérés comme un atout plutôt qu'un frein à la mise en culture. La couleur du sol est systématiquement observée, avec l'idée que «plus la terre est sombre, plus elle est riche en matière organique ». La structure grumeleuse est citée comme un paramètre indiquant la bonne qualité des sols, car cela démontre une bonne activité biologique et un sol «qui respire» (ferralitisols à drainage vertical libre).

Les agriculteurs ayant suivi des formations prêtent souvent plus attention aux indicateurs chimiques des sols, les plus importants et les mieux maîtrisés étant la matière organique (MO) et le $\mathrm{pH}$. L'amendement calcique est réalisé par $92 \%$ des agriculteurs. Seuls $7 \%$ ne gèrent pas la fertilité de manière chimique ou organique (Tab. 2).

Enfin, la profondeur utile du sol n'est pas considérée comme un élément important. Les agriculteurs estiment que leurs sols sont profonds (sauf présence de nappe dans les sols hydromorphes) et homogènes.

Ces perceptions générales et l'intérêt porté à ces variables par les agriculteurs varient pour chaque groupe, en fonction notamment de leur objectif de production.

Dans le cadre des systèmes de production à forts intrants, les agriculteurs considèrent qu'un «bon » sol est productif, leur indicateur est donc le rendement qui est un critère subjectif la plupart du temps car il n'est pas mesuré. La pratique avancée comme solution à leurs sols peu fertiles est l'utilisation de fertilisant. Ils se déclarent désintéressés par la question de la qualité des sols. Le sol est alors plus un support qu'une ressource. C'est d'ailleurs ce groupe qui considère les conditions pédoclimatiques comme un frein léger à la mise en culture (Tab. 1).

Dans le cadre des systèmes de production à faible impact, les agriculteurs estiment qu'un «bon» sol est productif et le voient comme un objet vivant nécessaire à comprendre. Leurs indicateurs sont variés et souvent liés à l'aspect général du sol (texture, couleur, pierrosité, parfois structure, conditions hydrodynamiques [présence ou absence de phases inondées ou à l'inverse de sécheresses, capacités drainantes]). Certains citent le $\mathrm{pH}$ et les analyses de sol. Ils font appel à des pratiques variées et combinées.

Dans le cadre des systèmes de production traditionnels, les agriculteurs observent la quantité et la qualité des produits récoltés pour déterminer si le sol est «bon». Ils se basent tous sur la couleur et la texture pour décrire leur sol et parfois sur la quantité d'adventices qui déterminent le moment où il va falloir changer de parcelle dans une dynamique d'abattis itinérant. Ils disent mobiliser un ensemble de connaissances transmises par leurs ancêtres (buttes, paillis, brûlis, jachère) pour garantir la qualité de leurs terres sur le long terme. 
Tableau 3. Stocks de carbone $\left(\mathrm{tC}_{\mathrm{C}} \mathrm{ha}^{-1}\right)$ des sols agricoles et variations par rapport à la référence sous forêt ( $n=31$ parcelles), couche $0-0,5 \mathrm{~m}$, en fonction du temps et du mode de culture.

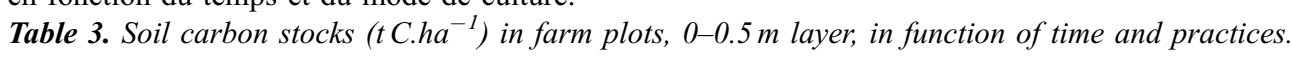

\begin{tabular}{lllllll}
\hline $\begin{array}{l}\text { Stock } \\
\text { agricole }\end{array}$ & $\begin{array}{l}\text { Stock } \\
\text { forestier }\end{array}$ & Delta & Stock & Delta & GES (teq & Temps depuis mise \\
$\left.\mathrm{CO}_{2} \cdot \mathrm{ha}^{-1} \cdot \mathrm{an}^{-1}\right)$ & Information & Type de sol \\
\hline
\end{tabular}

\begin{tabular}{|c|c|c|c|c|c|}
\hline \multicolumn{6}{|c|}{ Systèmes à forts intrants } \\
\hline 100,05 & 102,55 & $-2,5$ & $-2,44$ & 4,5 & 20 \\
\hline 36,92 & 70,34 & $-33,42$ & $-47,51$ & 6,3 & 8 \\
\hline 37,92 & 70,34 & $-32,42$ & $-46,09$ & 6,3 & 8 \\
\hline 29,68 & 73,02 & $-43,34$ & $-59,35$ & nd & 30 \\
\hline 143,21 & 161,34 & $-18,13$ & 11,24 & nd & 5 \\
\hline
\end{tabular}

\section{Systèmes à faible impact}

\section{Maraîchage}

$\begin{array}{crrrlr}206,85 & 128,97 & 77,88 & 60,39 & 2,5 & 5 \\ & & & & & \\ 123,45 & 128,97 & -5,52 & -4,28 & 2,5 & 2 \\ 114,60 & 121,91 & -7,31 & -6,00 & 3 & 15 \\ 204,79 & 126,45 & 78,34 & 61,95 & 1 & 4 \\ 90,88 & 80,82 & 10,06 & 12,45 & 1 & 3 \\ 241,4 & 201,09 & 40,31 & 20,05 & 1 & 3 \\ 101,3 & 126,45 & -25,15 & -19,89 & 1,4 & 10 \\ 126,18 & 126,45 & -0,27 & -0,21 & 1,4 & 10 \\ 414,47 & 371,31 & 43,16 & 11,62 & 1,7 & 5 \\ & & & & & \\ 425,33 & 371,31 & 54,02 & 14,55 & 1,7 & 5\end{array}$

\section{Arboriculture}

$\begin{array}{crrrlr}125,77 & 126,45 & -0,68 & -0,54 & 3 & 4 \\ 82,77 & 111,26 & -28,49 & -25,61 & 2 & 20 \\ 122,4 & 121,91 & 0,49 & 0,40 & 1 & 10 \\ 124,8 & 121,91 & 2,89 & 2,37 & 3 & 20 \\ 69,82 & 112,00 & -42,8 & -37,66 & 0,3 & 6 \\ 123,79 & 112,00 & 11,79 & 10,53 & 0,3 & 6 \\ 56,9 & 71,61 & -14,71 & -20,54 & 0,3 & 2 \\ 82,88 & 51,32 & 31,56 & 61,50 & \text { nd } & 30\end{array}$

Station expérimentale : Combi, grandes cultures et prairies (Fujisaki et al., 2014)

\begin{tabular}{|c|c|c|c|c|}
\hline 77,7 & 73,2 & 4,5 & 6,15 & nd \\
\hline 70,5 & 73,2 & $-2,7$ & $-3,69$ & nd \\
\hline 64,1 & 73,2 & $-9,1$ & $-12,43$ & nd \\
\hline
\end{tabular}

Systèmes traditionnels

\begin{tabular}{rrrrlll}
93,47 & 94,00 & $-0,53$ & $-0,56$ & nd & 2 & Changement de parcelle tous les 3 ans \\
51,95 & 78,91 & $-26,96$ & $-34,17$ & nd & 30 & Abattis \\
61,84 & 65,26 & $-3,42$ & $-5,24$ & nd & 30 & Abattis \\
93,69 & 87,07 & 6,62 & 7,60 & nd & 30 & Abattis \\
63,56 & 73,21 & $-9,65$ & $-13,18$ & nd & 30 & Abattis \\
68,31 & 60,25 & 8,06 & 13,38 & nd & 15 & Abattis \\
\hline
\end{tabular}

Reprise de la parcelle après une jachère de 2 ans pour du maraîchage monospécifique, rotations

Maraîchage sous serre, monospécifique

Maraîchage sous serre, monospécifique

Maraîchage monospécifique

Arboriculture monospécifique

Ferallitisol

Redoxisol

Redoxisol

Thalassosol

Ferallitisol

Culture sous serre, sur terrasse, BRF, rotations

Culture sur terrasse, rotations

Buttes, rotations et associations

Buttes, BRF, rotations et associations

Buttes, BRF, rotations et associations

Buttes, BRF, rotations et associations

Buttes avec paillis récent, rotations

Buttes avec paillis décomposé,

rotations

Planches avec des apports conséquents de matière (BRF et fumier), rotations Planches avec des apports conséquents de matière (BRF et compost), rotations

Agro-forêt
Monospécifique
Monospécifique
Monospécifique
Inter-rang, Monospécifique
Rang, Monospécifique
Monospécifique
Agro-forêt

Ferallitisol

Ferallitisol

Ferallitisol

Ferallitisol

Ferallitisol

Ferallitisol

Podzosol

Redoxisol

Prairie

Culture de céréales

Semi direct de céréales

Ferallitisol

Ferallitisol

Ferallitisol

Ferallitisol

Ferallitisol

Ferallitisol

Ferallitisol

Ferallitisol

Ferallitisol

Les buttes sont reconstituées tous les ans ou tous les 3 ans, ce qui homogénéise les apports de matière organique sur l'ensemble de la parcelle. 
Le labour est limité aux 30 premiers centimètres de profondeur.

Dans les enquêtes, le facteur sol est apparu déterminant. Dans la gestion spatiale du système de production, nous observons que l'agriculteur s'adapte aux spécificités des sols par le choix des cultures et des pratiques. Par ce biais, l'objectif est bien entendu d'optimiser la production en bénéficiant des «potentialités naturelles » du sol. Ainsi, concernant la couleur du sol, il apparait que $90 \%$ des agriculteurs y sont sensibles et mettent donc indirectement la teneur en MO en avant, clé du maintien de la fertilité des sols.

\subsection{Effet des pratiques sur le carbone du sol}

Pour la couche $0-0,5 \mathrm{~m}$, on observe une grande diversité de valeurs du stock organique de carbone dans les parcelles agricoles (Tab. 3), de 30 à $425 \mathrm{tC} \cdot \mathrm{ha}^{-1}$, tout comme leurs références sous forêt, où les $\mathrm{SOC}$ sont compris entre 51 et $371 \mathrm{tC}^{\mathrm{h}} \mathrm{ha}^{-1}$. Les SOC agricoles les plus élevés correspondent à des sols cultivés sur des sols aux SOC naturellement élevés sous forêt, dont la défriche récente est associée à l'utilisation de bois raméal fragmenté (paillis constitué grâce au broyage des bois de défriche). A contrario, les déstockages les plus importants sont représentés par des parcelles en maraîchage avec utilisation d'intrants chimiques ou par l'arboriculture à faible impact. Cela est lié à deux facteurs : la nature sableuse des sols (podzosols et fluvisols) et des teneurs en matière organique et éléments minéraux assez réduites ou un apport de matière organique limité. En effet, la diminution des entrées de carbone du sol est ici également déterminante, comme observé par Fujisaki et al. (2018) sur la station expérimentale de Combi.

Dans les systèmes de production à faible impact, la tendance est à l'accroissement des stocks avec le temps. En effet, lorsque la défriche et les pratiques sont à faible impact, les parcelles étudiées se rapprochent de leur niveau de stock initial, même à très court terme. Sur les 10 systèmes maraîchers à faible impact étudiés, les 6 qui utilisent le bois raméal fragmenté ont dépassé le niveau de stock initial. On peut alors se poser la question des transferts de fertilité par les amendements organiques réalisés. Ces résultats doivent être mis en perspective avec les autres travaux amazoniens aux variations de stocks hétérogènes allant de +20 à $-40 \%$ après mise en culture (Fujisaki et al., 2015a). Il existe encore trop peu de données pour en déduire une règle stricte en dehors des systèmes pérennes à pâturages pour le bassin amazonien.

Les premiers bilans de gaz à effet de serre des exploitations montrent, en fonction des variations du stock organique de carbone, que les systèmes de production à forts intrants ont plutôt une tendance au déstockage de carbone et sont la forme d'agriculture la plus émettrice (Tab. 3). Les résultats pour l'arboriculture sont variables, mais les bilans d'émission sont très faibles. S'agissant du maraîchage à faible impact, les émissions se situent entre 1 et 3 teq $\mathrm{CO}_{2} \cdot$ ha $^{-} 1 . \mathrm{an}^{-1}$ avec une grande variabilité du stock organique de carbone (liée au nombre d'années de culture et à l'effet de la valeur du stock de carbone de référence initial). Dans l'ensemble, l'usage des buttes ou de terrasses permet un accroissement considérable des SOC entre 12 et $62 \%(0-0,5 \mathrm{~m}$ de sol), et ce en un laps de temps très court (moins de $5 \mathrm{ans}$ ). Cette observation est encourageante, car elle entre dans le principe du $4 \%$ (Derrien et al., 2016), rappelant que l'agriculture n'est pas forcément émettrice de gaz à effet de serre, mais peut au contraire participer à la séquestration du carbone. Toutefois, ces effets sur le stock ne sont pas pérennes du fait de la disparition des fragments de bois, ce qui est inéluctable dans ces conditions climatiques (Perrin et al., 2014; Fujisaki et al., 2015b).

\section{Conclusion}

Nous avons identifié 3 systèmes de production caractérisés par des objectifs de production et de pratiques qui mettent en valeur quelques originalités. L'analyse des freins au développement de l'activité agricole et celle de la perception des sols par les agriculteurs montrent que «l'objet sol» est un indicateur reconnu qui conditionne les modes de gestion de l'espace.

Les stocks de carbone organique des sols sous végétation naturelle sont élevés et varient rapidement et fortement après la défriche et la mise en culture. Les mesures chez les agriculteurs sont cohérentes et soulignent bien la fragilité des systèmes solplante dès lors que l'on défriche. Nous avons vu en particulier que les phénomènes sont rapides, tant sur la réactivité à constituer des stocks de carbone exemplaires avec certaines pratiques, qu'à les voir se dégrader dans certains systèmes intensifs. Si l'impact des pratiques à forts intrants se traduit par des baisses drastiques du stock organique de carbone, jusqu'à $-50 \%$ du SOC à $\mathrm{T}_{0}$ en 5 ans, les pratiques à faible impact peuvent avoir des effets bénéfiques sur les dynamiques de stockage du carbone, en plus d'avoir un impact environnemental réduit.

Nous soulignons en particulier le caractère exploratoire des bilans des émissions des exploitations que nous avons effectués. Ces premiers résultats montrent que les émissions sont les plus élevées dans les sites à forts intrants. Notre étude montre qu'il est possible d'avancer dans le secteur des terres entre carbone environnemental et carbone agricole et qu'il est possible de mettre en place des solutions durables. La Guyane s'inscrit aussi bien dans les préoccupations des pays en développement que du Nord, qui insistent depuis la Cop 17 de 2011 sur l'adaptation et l'atténuation. Quels que soient les choix de transformation de la forêt et des sols, cela reste un enjeu.

\section{Références}

Baveye PC, Baveye J, Gowdy J. 2016. Soil “Ecosystem” Services and Natural Capital: Critical Appraisal of Research on Uncertain Ground. Front. Environ. Sci. 4. DOI: 10.3389/fenvs.2016.00041.

Cochet H, Devienne S. 2006. Fonctionnement et performances économiques des systèmes de production agricole : une démarche à l'échelle régionale. Cahiers Agricultures 15: 578-583. DOI: 10.1684/agr.2006.0028.

Dallaporta D, Bochu JL, Vigne M, Ouliac B, Zoogones LJ, Lecomte P, et al. 2016. Taking into account carbon sequestration of pasture in carbon balance of cattle ranching systems established after deforestation in Amazonia. In: Proc. 10th Int. Rangeland Congress, Saskatoon, Canada, 6-22 July 2016, pp. 399-400.

Derrien D, Dignac MF, Basile-Doelsch I, Barot S, Cécillon L, Chenu C, et al. 2016. Stocker du C dans les sols: quels mécanismes, 
quelles pratiques agricoles, quels indicateurs? Etude et Gestion des Sols 23: 193-224.

FAO. 2015. Rapport sur l'État des ressources en sols dans le monde. Rome, Italie : FAO. http://www.fao.org/3/a-i5126f.pdf.

FAO. 2018. Carbone organique du sol : Une richesse invisible. Rome, Italie: FAO.

FAO. 2019. Measuring and modelling soil carbon stocks and stock changes in livestock production systems: Guidelines for assessment (Version 1) Livestock Environmental Assessment and Performance (LEAP) partnership. Rome, Italie: FAO, $170 \mathrm{p}$.

Fujisaki K. 2014. Devenir des stocks de carbone organique des sols après déforestation et mise en culture : une analyse diachronique en contexte amazonien. Thèse Univ Supagro Montpellier, France, $237 \mathrm{p}$.

Fujisaki K, Perrin AS, Desjardins T, Bernoux M, Balbino LC, Brossard M. 2015a. From forest to cropland and pasture systems: a critical review of soil organic carbon stocks changes in Amazonia. Global Change Biology 21: 2773-2786. DOI: 10.1111/gcb.12906.

Fujisaki K, Perrin AS, Boussafir M, Gogo S, Sarrazin M, Brossard M. 2015b. Decomposition kinetics and organic geochemistry of woody debris in a ferralsol in a humid tropical climate. European Journal of Soil Science 66: 876-885. DOI: 10.1111/ejss.12277.

Fujisaki K, Chevallier T, Chapuis-Lardy L, Albrecht A, Razafimbelo T, Masse D, et al. 2018. Soil carbon stock changes in tropical croplands are mainly driven by carbon inputs: A synthesis. Agriculture, Ecosystems and Environment 259: 147-158. DOI: 10.1016/j.agee.2017.12.008.

GEC. 2016. Secteur UTCF : utilisation des terres leurs changements et la forêt. Méthodologie d'inventaire de gaz à Effet de serre. Cayenne, France: GEC.

GIEC. 2019. Climate Change and Land: an IPCC special report on climate change, desertification, land degradation, sustainable land management, food security, and greenhouse gas fluxes in terrestrial ecosystems. In Shukla PR, Skea J, Calvo Buendia E, MassonDelmotte V, Pörtner HO, et al., eds. Intergovernmental panel on climate change. https://www.ipcc.ch/srccl/.

Grandisson M. 1997. Gestion des systèmes d'agriculture itinérante sur brûlis dans l'ouest Guyane : contribution à l'étude de la reproductibilité et de la fertilité. Thèse Univ. Antilles-Guyane, France, 258 p.

IEDOM. 2019. Rapport annuel 2018 de l'IEDOM. Paris, France: Institut d'émission des départements d'outre-mer, $185 \mathrm{p}$.

Imbert G. 2010. L'entretien semi-directif: à la frontière de la santé publique et de l'anthropologie. Recherche en soins infirmiers 102 (3): 23-34. DOI: 10.3917/rsi.102.0023.
INSEE. 2018. Recensement de la population en Guyane: la démographie guyanaise toujours aussi dynamique. Insee Analyses Guyane 27. https://www.insee.fr/fr/statistiques/3309060.

INSEE. 2019. Le bilan économique 2018: L'économie guyanaise redémarre. Insee Conjoncture Guyane 5: 48.

Lal R, Follett RF, Stewart BA, Kimble JM. 2007. Soil carbon sequestration to mitigate climate change and advance food security. Soil science 172(12): 943-956. DOI: 10.1097/ ss.0b013e31815cc498.

Landais E. 1998. Modelling farm diversity: new approaches to typology building in France. Agricultural Systems 58(4): 505-527. DOI: 10.1016/S0308-521X(98)00065-1.

McBratney A, Field DJ, Koch A. 2014. The dimensions of soil security. Geoderma 213: 203-213. DOI: 10.1016/j.geo derma.2013.08.013.

Olivier de Sardan JP. 1995a. Anthropologie et développement : essai en socio-anthropologie du changement social. Collection "Hommes et sociétés". Paris, France: Karthala.

Olivier de Sardan JP. 1995b. La politique du terrain. Enquête 1: 71109. DOI: $10.4000 /$ enquete.263.

Ozier-Lafontaine H, Galan M, Reinette Y, Gelin S, Hernandez P, Jacolot $\mathrm{P}$, et al. 2018. Transition agroécologique des productions végétales en Guyane: Quel dispositif pour assurer la multiperformance et la résilience des systèmes agricoles? Innovations Agronomiques, INRA, 64: 113-132. DOI: 10.15454/ $1.5408028469930225 \mathrm{E} 12$.

Perrin AS, Fujisaki K, Petitjean C, Sarrazin M, Godet M, Garric B, et al. 2014. Conversion of forest to agriculture in Amazonia with the chop-and-mulch method: Does it improve the soil carbon stock? Agriculture, Ecosystems \& Environment 184: 101-114. DOI: 10.1016/j.agee.2013.11.009.

Piantoni F. 2009. La question migratoire en Guyane française. Hommes \& migrations 1278: 198-216. DOI: 10.4000/hommesmi grations. 259

Razafimbelo TM, Andriamananjara A, Rafolisy T, Razakamanarivo $\mathrm{H}$, Masse D, Blanchart E, et al. 2018. Impact de l'agriculture climato-intelligente sur les stocks de carbone organique du sol à Madagascar. Cahiers Agricultures 27(3): 35001. DOI: 10.1051/ cagri/2018017.

Renoux F, Fleury M, Reinette Y, Grenand P, Grenand F. 2003. Slash and burn agriculture in the Maroni and Oyapock river basins: Dynamics and adaptation to spatial constraint. Rev. For. Fr. 55: 236-259.

Citation de l'article : Courte A, Cialdella N, Muller A, Blanfort V, Bochu J-L, Brossard M. 2020. Recenser et évaluer les pratiques agricoles qui stockent le carbone des sols, premier pas vers une agriculture à faible impact en Guyane. Cah. Agric. 29: 21. 